\title{
La capacité en droit, un diplôme réhabilité grâce à la formation ouverte et à distance ?
}

\author{
Emilie Bomal* - Thomas Zacher* \\ Genevieve Thomas-Debenest**_ Françoise Galland* \\ * Service des Technologies de l'Information et de la Communication (STIC) \\ ** UFR de Droit, Economie et Gestion \\ Université d'Angers - 2 Bd Lavoisier - F-49000 - Angers \\ francoise.galland@univ-angers.fr
}

\begin{abstract}
RÉSUMÉ. Alors que les universités françaises sont les seules habilitées à dispenser la capacité en droit, la majorité a abandonné cette formation, notamment en raison du fort taux d'abandon des inscrits. C'est pourquoi nous avons été amenés à mettre en place un dispositif de formation ouverte et à distance qui a nécessité un remaniement total de la formation. Ce changement a été possible grâce au soutien institutionnel de la région (financement de la production des ressources) et de l'université (fonctionnement du dispositif et politique d'incitation auprès des enseignants-chercheurs). Le dispositif s'articule autour de trois axes : modularité, alternance présenceldistance et pédagogie différenciée. Le bilan des 2 premières années de fonctionnement est très encourageant. Il reste à pérenniser le dispositif et mettre en place des indicateurs pour suivre cette expérience que nous avons vécue comme une révolution socio-économique et institutionnelle.

ABSTRACT. Although only French universities are allowed to dispense the basic certificate in law, most of them have abandoned this diploma, particularly because of the high level of withdrawal among the registered students. That's why we have been led to setting up a distance learning course which has required a total reorganization of the training course. It has been possible thanks to a political will both of the Region (financing of the creation of learning contents) and the University (functioning of the course and incentive policy for the teachers and lecturers). The training course is structured along three main lines: adaptability, alternation of on-campus and distance learning and flexible educational methods. The appraisal of the first two years of running shows that there's a clear improvement. Now, we have to ensure a perennial course with good indicators to follow this socio-economic and institutional framework which, for us, looks like a revolution in common practice.

MOTS-CLÉS : formation à distance, diplôme, capacité en droit.

KEYWORDS: e-learning, diploma, certificate in law.
\end{abstract}

DOI:10.3166/D\&S.5.53-66@Cned/Lavoisier

D\&S - 5/2007. Industrialisation, repérages, pages 53 à 66 


\section{Problématique}

En France, l'accès à l'université passe traditionnellement par le baccalauréat. Pour permettre aux non bacheliers français d'intégrer l'université, deux diplômes leur sont encore offerts : le DAEU et la capacité en droit. Le certificat de capacité en droit est autant un outil d'ascension sociale qu'un outil d'enrichissement personnel; outil d'ascension sociale tout d'abord car, en plus de l'accès à l'université (en particulier le DEUG de Droit), il offre la possibilité de se présenter à certains concours; outil d'enrichissement personnel ensuite dans la mesure où il permet d'acquérir des connaissances juridiques de base, nécessaires aussi bien dans la vie quotidienne que dans toute vie professionnelle. Alors que les Universités françaises sont les seules habilitées à dispenser ces diplômes, la majorité d'entre elles ont abandonné la formation à la capacité en droit, créée en 1804, notamment en raison du fort taux d'abandon lié aux candidats souvent en situation d'échec scolaire ou professionnel. L'Université d'Angers n'a pas échappé à ce désintérêt : 39 inscrits en première année en 2002-2003 et 2 en deuxième année. Une réflexion a alors été engagée pour étudier les causes de ces abandons et proposer une solution innovante pour que nos apprenants puissent bénéficier d'une offre de formation adaptée à leur situation, d'autant que notre établissement restait le seul à dispenser cette formation sur un périmètre de plusieurs départements. Résumée en une phrase, la problématique était simple: supprimer la formation en l'état ou essayer une autre formule?

\section{Gestion du projet}

Notre démarche a donc consisté à faire au préalable une étude de l'existant (public, modalités de la formation, interactions entre enseignants et apprenants, taux d'abandon, taux de réussite). Ce bilan préliminaire, coordonné par la cellule TICE de notre établissement, le Service des Technologies de l'Information et la Communication (STIC), a été réalisé avec l'ensemble des partenaires impliqués par ce diplôme : les apprenants par le biais d'une enquête, la responsable pédagogique, les enseignants et chargés de TD sans oublier une personne socialement très impliquée dans ce dispositif, la secrétaire chargée des inscriptions et du suivi des apprenants.

A l'issue de ce constat, nous avons décidé de mettre en place un dispositif de formation ouverte et à distance (DFOAD). Nous envisagerons d'abord la conception du dispositif : ressources pédagogiques, choix des activités pédagogiques, répartition présentiel/distance, accompagnement des apprenants, gestion des examens... Enfin, nous présenterons, avec un recul encore restreint, le suivi de la formation à l'issue de 2 ans de fonctionnement pour la première année et 1 an pour la deuxième année.

\section{I - Etude de l'existant}

Si le diplôme, étalé sur deux ans, concerne un public peu contraint comme les inactifs et les retraités intéressés par le droit, il s'adresse aussi et surtout aux jeunes 
en situation d'échec scolaire, peu autonomes, aux personnes en situation précaire et/ou en recherche d'emploi. Cette dernière catégorie impose à l'équipe enseignante, consciente de ces impératifs, des contraintes lourdes puisqu'ils doivent être libérés dans la journée pour rechercher un travail. C'est pourquoi les cours étaient dispensés en soirée - à raison de trois soirs par semaine, ou le samedi matin - sur un même lieu (pour des contraintes économiques) à l'Université d'Angers.

Dans le contexte universitaire de la formation, la capacité est peu valorisée au sein des enseignants - chercheurs qui trouvent naturellement plus d'attrait à s'impliquer dans des formations touchant le cœur de leur public: les étudiants bacheliers. De ce fait, les enseignants sont généralement rarement motivés d'autant que les horaires sont peu attractifs. En revanche, ceux qui s'impliquent ont un esprit militant et cette attitude volontariste s'est traduite par un excellent contact entre étudiants et enseignants en enseignement présentiel.

Les disciplines enseignées concernent différents secteurs du droit et les modules sont dispensés en parallèle tout au long de l'année avec un examen final (2 sessions) en fin de chaque année.

Au cours de l'année universitaire 2002 - 2003, l'effectif comportait en $1^{\mathrm{e}}$ année : 40 inscrits, 20 présents à l'examen, 6 admis ; en $2^{\mathrm{e}}$ année : 7 inscrits et présents à l'examen, 4 admis. Au cours des années antérieures, les chiffres avaient même été certaines années encore plus faibles ! Quant à l'effectif initial restreint, il intéressait un bassin géographique théorique de plusieurs départements puisque sur les 3 universités de la région, celle du Mans avait déjà fermé cette formation.

Nous avons donc procédé à une enquête par questionnaire envoyé aux 40 inscrits à la rentrée 2002. L'échantillon est bien sûr restreint et les non-répondants (17) auraient probablement été particulièrement intéressants. Celle-ci a montré que l'abandon des apprenants était essentiellement lié aux contraintes horaires et géographiques. Ces difficultés étaient le plus souvent d'ordre familial, financier, professionnel, psychologique (situation d'échec) concernant un public généralement peu autonome. Pour pallier ces contraintes spatio-temporelles, certains apprenants, plus autonomes, avaient déjà amorcé une solution en communiquant avec d'autres apprenants par courriel pour rattraper des cours, se les faire expliquer et échanger sur leur méthode de travail.

Au cours de cette enquête préliminaire, nous avons également testé auprès de ces anciens apprenants le projet de DFOAD. Globalement ils ont été assez favorables au projet mais certaines inquiétudes ont été soulignées telles que la peur de perdre tout contact -avec les enseignants et les autres apprenants- et la désynchronisation entre l'enseignement et l'apprentissage. Certains apprenants ont émis le souhait d'avoir une formule qui se situerait à mi-chemin entre le «tout » présentiel et le «tout» à distance. Contrairement à des idées reçues, ce n'est pas l'appréhension de l'outil informatique qui est ressorti comme principal obstacle à la mise en ligne de la formation. 


\section{II - Mise en place et fonctionnement du dispositif de FOAD}

Face à ces constats, la responsable pédagogique de la formation a accepté en 2001 de «mettre à plat» cette formation pour lui donner un nouveau souffle et tenter de pérenniser cette formation historique, symbole des missions principales de nos universités.

La mise en oeuvre du projet, véritable « révolution pédagogique », a été possible :

- grâce à une forte implication de l'équipe enseignante, particulièrement sensible au maintien d'un tel diplôme, puissant outil d'ascension sociale (y compris pour certains enseignants participant maintenant à cet enseignement),

- grâce aussi à une forte volonté de notre université soucieuse d'adapter ses formations à un public d'apprenants en difficulté,

- grâce enfin à une autre forte volonté politique régionale convaincue de la nécessité de diversifier l'offre de formation (Université Virtuelle en Pays de la Loire - UVPL).

Cette part de volontarisme est au niveau régional et local bien antérieure à la mise en place de la capacité en droit puisqu'elle remonte à la création de l'UVPL en 2000, décidée conjointement entre le Conseil Régional, les 3 universités ligériennes (Nantes, Angers, le Mans) et l'IUFM.

Cette création résultait d'un double constat :

- un taux de bacheliers intégrant l'enseignement supérieur inférieur à la moyenne nationale. Ce constat a été traduit comme une offre de formation insuffisante et/ou inadaptée,

- un taux d'abandon en $3^{\mathrm{e}}$ cycle supérieur à la moyenne nationale, ce qui pour la région se traduisait par un manque de création de PME-PMI nuisible à l'économie de la région.

Pour ces 2 raisons, l'UVPL a été créée. Le budget annuel de 1,5 M $€$ a été consacré, entre autres, à la mise en place de formations dédiées à un public «nouveau ». Compte-tenu des résultats de l'enquête (problème de distance et de temps) la capacité en droit s'inscrivait typiquement dans cette logique et c'est la raison pour laquelle nous avons proposé aux enseignants de mettre en place un DFOAD.

La concrétisation de ce projet est le fruit d'une étroite collaboration entre une équipe enseignante soudée autour d'une responsable pédagogique convaincue et une équipe technico-pédagogique, le STIC, service commun de l'université créé en 2000, avec pour mission principale l'aide à la pédagogie et particulièrement sensible à ce public en difficulté, sans oublier le soutien logistique et financier très significatif de la région. 
La conception du dispositif est essentiellement financée par la subvention régionale. Elle est dédiée à la rémunération des enseignants pour produire les ressources et concevoir les activités pédagogiques, au STIC pour la médiatisation et l'ingénierie pédagogique. Pour la conception de la $1^{\mathrm{e}}$ année, il a été convenu avec les enseignants que leur rémunération serait étalée sur 2 ans afin de permettre des ajustements à l'issue de la première année de fonctionnement. Pour objectiver ces ajustements, une enquête a été programmée à l'issue du $1^{\mathrm{er}}$ examen auprès des apprenants et des enseignants.

En revanche, l'Université prend totalement à sa charge le fonctionnement du dispositif en ligne. Les activités de tutorat pédagogique, assurées par les enseignants, sont reconnues au même titre qu'elles l'auraient été en présentiel (heures TD). Cette prise en compte est formalisée par un document commun voté par les conseils d'administrations des 4 établissements partenaires de l'UVPL. Les recettes obtenues avec les inscriptions restent modestes puisque les frais d'inscription sont restés les mêmes que ceux du présentiel.

$\mathrm{Au}$ vu des résultats encourageants obtenus pour la $1^{\mathrm{e}}$ année, l'UFR a décidé de soutenir l'équipe enseignante de la $2^{\mathrm{e}}$ année par la mise à disposition d'un Attaché Temporaire d'Enseignement et de Recherche pour les aider à concevoir des activités pédagogiques qui n'étaient pas prévues dans le cadre de l'enseignement présentiel.

Compte tenu des premiers résultats de l'enquête préliminaire, la solution d'un DFOAD, consistant à rendre accessible la formation en tout temps et en tout lieu par le biais des nouvelles technologies, s'est donc très vite imposée comme la réponse la mieux adaptée à ces nouveaux besoins. Ce type de dispositif trouve tout à fait sa place dans cette discipline en constante évolution car elle est étroitement liée à l'actualité. Après une année d'effort de conception, c'est à l'occasion de ses 30 ans d'existence à l'université d'Angers que la capacité en droit en ligne a pu accueillir, septembre 2003, sa première promotion.

La réalisation de ce dispositif a nécessité un remaniement total de la formation autant en terme d'organisation que de conception des contenus pédagogiques dans la mesure où celui-ci vient en substitution et non en complément de la formation en présentiel. La maquette de la formation a été intégralement repensée : scénarisation des contenus pédagogiques, définition et création des activités pédagogiques, formes du tutorat, articulations des modules ainsi que les modalités d'examen. Durant cette phase de ré-ingénierie pédagogique nous avons insisté sur l'importance de l'approche pédagogique pour que les enseignants ne se «focalisent» pas sur l'aspect technique de la médiatisation. Il a donc fallu démystifier et montrer les potentialités offertes par l'ordinateur car la majorité des enseignants, acteurs du projet, n'avaient pas de compétences en informatique et ne disposaient pas de connexion Internet à domicile. Cette étape a été l'occasion de les sensibiliser aux facteurs et aux conséquences induits par l'intégration des TIC dans leurs pratiques pédagogiques. En effet, dans ce nouveau rapport au savoir, l'enseignant devient un 
guide, un « coach », l'apprenant - placé au cœur du dispositif - devient acteur à part entière de sa formation.

Sur le plan pratique, ce travail s'est réalisé en plusieurs étapes. Une première initiation (le plus souvent) à l'informatique a été nécessaire... avant d'aborder la pédagogie proprement dite. Ensuite, la progression s'est faite par la mise en ligne des ressources, le domaine d'excellence des enseignants. Et, au cours de ces échanges, les ingénieurs pédagogiques ont pu progressivement les amener à concevoir le dispositif de FOAD, à définir les activités pédagogiques... La distance a été discutée au moment de la constitution de la maquette: choix, durée des moments de présentiel et de distance, activités tutorées...

Les enseignants sont passés en cours de conception et de $1^{\mathrm{e}}$ année de fonctionnement d'une attitude "maître des contenus et maître de la pédagogie » à «maître des contenus » sans maîtrise de la pédagogie à distance. Peu à peu, les ingénieurs pédagogiques se sont « imposés » avec le soutien de la responsable de la cellule TICE, elle-même enseignante, dans les rares cas de discordance touchant la pédagogie. Ces premières réticences vis-à-vis des ingénieurs pédagogiques a complètement disparu pour la mise en place de la $2^{\mathrm{e}}$ année : le climat de confiance instauré avec les enseignants de $1^{\mathrm{e}}$ année a suffi à lever cet obstacle auprès de ceux de $2^{\mathrm{e}}$ année.

Le dispositif élaboré en collaboration avec l'équipe enseignante s'articule autour de trois axes: la modularité, l'alternance présence/distance et la pédagogie différenciée.

La modularité est la première transformation résultant de la mise en ligne de la capacité en droit. Elle se traduit par la création de modules indépendants et capitalisables permettant aux apprenants de pouvoir valider l'ensemble de la capacité en plusieurs années et, ainsi, de répartir leur charge de travail. Plus concrètement la première année de la capacité est composée de 3 modules successifs de $60 \mathrm{~h}$ répartis sur 10 semaines chacun. La deuxième année propose une organisation un peu différente dans la mesure où les apprenants travailleront simultanément sur 2 modules de $30 \mathrm{~h}$ répartis sur 10 semaines chacun. Cette démarche est un élément fort pour la formation professionnelle notamment dans les administrations, les collectivités et les entreprises. Il facilite en effet l'inscription d'apprenants intéressés seulement par un ou quelques modules de droit et qui sont prêts à s'investir, sur un délai court, pour suivre cette partie de la formation en plus de leur activité principale.

Le deuxième axe se concrétise par l'alternance des phases de regroupements en présentiel à l'université et des phases de travail à distance. Les périodes de regroupements constituent des périodes charnières (séminaire de rentrée, séances de régulation et de synthèse, examens). Le séminaire de rentrée, organisé sur deux jours, permet aux apprenants d'avoir une présentation des enseignements par les enseignants, d'obtenir des explications complémentaires sur le déroulement de la formation et des examens et de bénéficier d'une formation technique à l'utilisation 
de la plate-forme de formation utilisée dans le cadre du dispositif. Cette première prise de contact s'est rapidement avérée essentielle pour impulser une dynamique de groupe. Les séances de régulation et de synthèse - programmées en milieu de module soit au bout de cinq semaines- animées par les enseignants et les tuteurs (doctorants ou ATER) sont l'occasion de re-dynamiser les apprenants en apportant notamment des solutions à leurs problèmes méthodologiques ou de compréhension du cours. La deuxième modification induite par la mise en ligne de la capacité en droit est l'organisation des examens. Dans le cadre de l'enseignement en présentiel ceux-ci étaient uniquement organisés en fin d'année avec dans un premier temps les écrits et en cas d'admission les oraux. Dans le cadre du dispositif de FOAD une séance d'examen est prévue - écrits et oraux dans la même journée - après chaque module, soit 3 périodes d'examens par an, afin de motiver les apprenants à valider les modules suivants.

Le troisième axe se caractérise par la pédagogie différenciée qui se traduit notamment par la création d'activités pédagogiques et la mise en place d'un accompagnement pédagogique - ou tutorat - personnalisé. Nous avons accompagné l'équipe enseignante dans la scénarisation et la conception d'activités pédagogiques telles que des exercices auto-correctifs ou d'auto-évaluation (quizz, texte à trous, association d'éléments, ...) et des devoirs corrigés en tenant compte des exigences attendues aux examens. L'accompagnement des apprenants est double dans le sens où nous avons distingué le tutorat pédagogique, assuré par les enseignants concepteurs des modules, et le tutorat méthodologique, assuré par une doctorante qui a conçu - en collaboration avec les enseignants - des devoirs types et leurs corrigés. Le tutorat, pédagogique et méthodologique, est effectif durant les 10 semaines de chaque module. Celui-ci peut prendre différentes formes - forum, courriel ou téléphone - selon l'objectif visé. Le courriel est utilisé par les apprenants pour transmettre leur devoir au tuteur-doctorant qui devra par la suite leur retourner corrigé. Il peut également être utilisé pour un échange entre les apprenants mais il a été précisé au séminaire de rentrée que cet outil ne devait pas être utilisé à des fins pédagogiques. Autrement dit, toutes les questions d'ordre pédagogique doivent être posées dans le forum tout simplement parce qu'une question posée à un enseignant est susceptible, au même titre que la réponse, d'intéresser l'ensemble des apprenants. Nous souhaitons ainsi privilégier la mutualisation des échanges. Pour cela, nous avons créé un forum spécifique à chaque module. Par ailleurs, cinq autres forum ont été créés :

- un forum pour les échanges entre les enseignants et l'équipe du STIC,

- un forum pour toutes les questions d'ordre technique,

- un forum réservé aux échanges entre apprenants,

- et un forum réservé aux échanges entre enseignants.

D'une manière générale les échanges se font de manière asynchrone avec un délai de réponse précisé par les enseignants et sont principalement réactifs. 
Concernant le téléphone, nous devons préciser que nous n'avions pas proposé cet outil pour encourager les apprenants à se rendre sur la plate-forme de formation. Cependant, l'enseignant responsable du dernier module de la première année a souhaité, à titre expérimental, proposer cet outil... qui n'a obtenu aucun succès. Un apprenant a même expliqué qu'il préférait utiliser le courriel ou le forum parce que la formalisation écrite lui permettait d'une part, de mieux exprimer sa question et d'autre part, de mieux comprendre et mémoriser la réponse. Il y a eu, parallèlement, une appropriation du forum aussi bien côté enseignant que côté apprenant. Cette appropriation du forum est d'ailleurs très significative chez les enseignants qui au départ étaient sceptiques quant à l'interactivité que pouvait permettre l'usage du forum. Il s'est révélé que ces mêmes enseignants se sont rapidement pris au jeu : ils se sont même surpris à aller consulter le forum en dehors des plages horaires définies et même, une fois le module terminé.

Le forum réservé aux apprenants a également largement contribué à constituer cette communauté virtuelle entre apprenants, à l'image des contacts informels des étudiants en présentiel avant, après (et quelquefois pendant) les cours. Pour éviter toute déviance, l'équipe du STIC garde un regard sur ce forum et, bien évidemment, les apprenants sont prévenus de cette « surveillance » dès le séminaire de rentrée.

L'ouverture de la $1^{\mathrm{e}}$ année de la capacité à distance (2003) a été précédée d'une campagne de communication auprès des instances régionales par mailing (ANPE, mairies et Conseil général, points d'accès internet institutionnels), voie de presse, radio et TV locales, manifestations (journées portes ouvertes de l'Université). Les internautes étaient informés à partir du site portail de l'UVPL et, évidemment celui de l'université. Nous avons également utilisé des listes de diffusion comme celle de THOT/Cursus.

\section{III - Bilan des 2 premières années de fonctionnement}

Il y a une nette amélioration quantitative (au niveau des effectifs et des reçus) comme le montre le tableau ci-dessous. Il n'est à l'heure actuelle plus d'actualité de supprimer cette formation!

Cependant, ces résultats sont à interpréter avec précaution puisqu'ils concernent, actuellement, seulement 2 promotions pour la $1^{\mathrm{e}}$ année et 1 seule pour la $2^{\mathrm{e}}$.

Par ailleurs, si le niveau des apprenants est infiniment supérieur aux années précédentes, les résultats aux examens ne reflètent que partiellement cette amélioration de niveau. En effet, les résultats aux examens ne sont pas un indice suffisant pour apprécier la pertinence du dispositif car ils ne représentent l'objectif final que pour une partie des apprenants. Ainsi, sur les 84 inscrits en 2003, au moins 23 étaient, au minimum, titulaires d'un baccalauréat (et probablement plus car ce champ n'est pas obligatoirement documenté). Sur ces 84 inscrits, 32 ont 40 ans ou plus. 
Cette amélioration de niveau pourrait s'expliquer :

- soit par un biais de recrutement,

- soit par une modification du niveau d'exigence pour l'obtention du diplôme. Cet argument paraît peu probable puisque l'équipe enseignante (à une exception près) est restée la même et le programme n'a pas été modifié,

- soit par une évolution de la pédagogie, orientée suivant le mode plutôt transmissif en présentiel et évoluant avec l'accompagnement à distance vers un mode plus socio-constructiviste, peut-être mieux adapté à ce contexte.

\begin{tabular}{|c|c|c|c|c|c|c|c|c|c|c|c|c|}
\hline \multicolumn{13}{|c|}{ Inscriptions et Résultats aux examens } \\
\hline année & \multicolumn{4}{|c|}{$2002-2003$} & \multicolumn{4}{|c|}{ 2003-2004 } & \multicolumn{4}{|c|}{$2004-2005$} \\
\hline & \multicolumn{2}{|c|}{$1^{\mathrm{e}}$ Année } & \multicolumn{2}{|c|}{$2^{\mathrm{e}}$ Année } & \multicolumn{2}{|c|}{$1^{\mathrm{e}}$ Année } & \multicolumn{2}{|c|}{$2^{\mathrm{e}}$ Année } & \multicolumn{2}{|c|}{$1^{\mathrm{e}}$ Année } & \multicolumn{2}{|c|}{$2^{\mathrm{e}}$ Année } \\
\hline DFOAD & \multicolumn{2}{|c|}{ non } & \multicolumn{2}{|c|}{ non } & \multicolumn{2}{|c|}{ oui } & \multicolumn{2}{|c|}{ non } & \multicolumn{2}{|c|}{ oui } & \multicolumn{2}{|c|}{ oui } \\
\hline Inscrits & \multicolumn{2}{|c|}{40} & \multicolumn{2}{|c|}{6} & \multicolumn{2}{|c|}{84} & \multicolumn{2}{|c|}{6} & \multicolumn{2}{|c|}{73} & \multicolumn{2}{|c|}{23} \\
\hline \multicolumn{13}{|l|}{ Examen } \\
\hline session & 1 & 2 & 1 & 2 & 1 & 2 & 1 & 2 & 1 & 2 & 1 & 2 \\
\hline Inscrits & 17 & 8 & 5 & 2 & 43 & 12 & 5 & 0 & 46 & 10 & 17 & 5 \\
\hline Présents & 17 & 3 & 5 & 2 & 20 & 9 & 5 & 0 & 22 & 7 & 13 & 4 \\
\hline Admis & 6 & 0 & 3 & 1 & 10 & 4 & 3 & 0 & 16 & 6 & 12 & 2 \\
\hline $\begin{array}{c}\text { Total } \\
\text { Admis }\end{array}$ & \multicolumn{2}{|c|}{6} & \multicolumn{2}{|c|}{4} & \multicolumn{2}{|c|}{14} & \multicolumn{2}{|c|}{ J } & \multicolumn{2}{|c|}{22} & \multicolumn{2}{|c|}{14} \\
\hline
\end{tabular}

Grâce à la levée des contraintes spatio-temporelles, le recrutement s'est effectivement modifié. Le public se diversifie et la capacité répond à un besoin évident qui n'était plus assuré. En effet, en plus du public initial, on voit s'inscrire :

- Conseiller prud'homal,

- Femme d'origine étrangère et mariée, jeune, sans équivalence de diplôme, après un apprentissage du français,

- Parent en congé parental,

- Retraité,

- Secrétaire d'avocat,

- Titulaire d'une maîtrise autre que le droit et fonctionnaire de police ou Gendarmerie...

Par ailleurs, le bassin de recrutement a tendance à s'élargir. Outre les Pays de la Loire, il y a une attraction de la grande couronne parisienne (personnel des collectivités territoriales, par exemple). Sur les 84 inscrits en 2003, 27 proviennent 
du département, 12 de la région des Pays de la Loire (hors département), 38 du reste de la France, 7 de l'étranger.

L'enquête effectuée à l'issue de la première année a permis de relever quelques points importants.

L'accompagnement des apprenants en $1^{\mathrm{e}}$ année est essentiel pour qu'ils prennent confiance en eux. Dès qu'ils retrouvent cette assurance, les barrières tombent : ils communiquent entre eux et avec les enseignants

Côté enseignant, il est indispensable d'avoir une équipe soudée avec une bonne régularité des contacts. Le travail est lourd car l'enseignant doit rétablir autrement la spontanéité du cours en présentiel mais il est passionnant. Il doit anticiper le questionnement des apprenants, soit par des exemples pratiques, soit par des références à l'actualité, soit par des réflexions. Il faut aussi susciter des questions qui montrent que l'apprenant a, de lui-même, réfléchi et est en mesure de trouver une ou des réponses appropriées. Si on arrive à cette dernière étape, cela montre que la distance n'a pas altéré le présentiel et qu'elle peut même le dépasser.

Les contacts sont en effet très réels avec les apprenants grâce au forum. Ce constat est d'autant plus important que c'était la crainte majeure des enseignants !

Les regroupements présentiels doivent être l'occasion d'échanges privilégiés, en particulier au moment des examens où la tension et la fatigue des apprenants sont lourdes à supporter pour certains apprenants.

Comme pour tout enseignement, la mise à jour des ressources est une étape importante dans les disciplines enseignées. De plus, si la mise à jour des contenus s'accompagne d'illustrations basées sur des faits d'actualité, l'intérêt du cours sera décuplé ! L'été est la période la plus propice pour les enseignants à condition qu'ils disposent d'ordinateurs portables.

Sur le plan pratique, les apprenants sont motivés par cette nouvelle forme d'apprentissage. L'aspect technique n'a pas été considéré comme un obstacle, peutêtre aussi parce que le STIC assure une "quasi-hotline », en particulier, en début d'année (téléphonique et par courriel).

\section{Discussion}

Cette démarche, vécue comme une "révolution", résulte d'un ensemble de changements qui se sont «concrétisés» dans le dispositif de FOAD. En voici quelques arguments :

- le constat que l'apprenant est au cœur de la formation - s'il n'y a plus d'étudiant et s'il y a un besoin... - avec une meilleure prise en compte de ses difficultés !!!!

- la discipline : le droit, enseignement typiquement loin des TICE, 
- l'acceptation des enseignants d'un soutien de la part d'une cellule d'ingénierie pédagogique,

- l'obligation de travailler en équipe projet, d'accepter un retour, «un jugement »,

- des contraintes différentes par rapport au présentiel (peu de calendrier présentiel fixe mais un suivi rapproché - « sans absence longue »),

- une prise de conscience institutionnelle de l'enseignement hors présence étudiant,

- une reconnaissance «officielle» du rôle de tuteur social des personnels administratifs s'occupant de ce type de public,

- un constat de l'inadaptation des contraintes administratives d'inscription, des difficultés à mettre en place un examen adapté,

- un sentiment plus fort de travail d'équipe de la part des enseignants. Si chaque enseignant prend soin de respecter les règles établies (délai de réponse, modalités...) et sent que ses collègues en font autant, la classe virtuelle garde un climat serein. Si un enseignant ne respecte pas le contrat, le climat se dégrade et risque de nuire à l'ensemble de l'équipe enseignante.

Pour l'ensemble de ces points, nous avons essayé de trouver sur le terrain des solutions adéquates. Il est évident que cette expérience est trop récente pour considérer que nous maîtrisons le processus.

Parmi les points qui nous semblent particulièrement importants à investiguer dans les années à venir, nous insisterons sur 4 sujets: l'importance de l'accompagnement des apprenants, la pérennité du dispositif, l'analyse du public impliqué (et exclu) dans le dispositif, la transférabilité du dispositif à d'autres diplômes.

En ce qui concerne l'accompagnement des apprenants, nous avons pris conseil auprès des équipes de l'ENST et de l'UTC avec qui nous avons dans les mêmes moments achetés et modulés 3 formations-accompagnement «chef de projet de DFOAD», «conception de DFOAD» et «accompagnement de DFOAD ». Ces formations, initialement issues du campus numérique FIPFOD, ont été aménagées et adaptées à notre contexte universitaire. Et nous les utilisons maintenant pour former les enseignants et les personnels IATOS destinés à cet encadrement.

Nous avons utilisé, en matière d'accompagnement des apprenants, quasiment toutes les potentialités de la plate-forme (agenda, trombinoscope, forum, courriel...). Il y avait bien sûr des forums pédagogiques mais aussi un forum réservé aux étudiants (auquel la cellule TICE avait accès). Il a largement joué le rôle de "défouloir », de rapprochement pour un travail entre apprenants, de soutien moral pour éviter les découragements et abandons. Il nous a également servi à déclencher la sonnette d'alarme lorsqu'un enseignant ne remplissait pas son contrat (non réponse, réponse trop tardive, ton inapproprié) en transitant par le responsable pédagogique de la formation. Les enseignants prennent maintenant un peu plus 
d'assurance. Ils commencent à envisager une approche par étude de cas avec discussion, réflexion... Ce sera probablement aussi à terme l'occasion de mutualisation et de collaborations interdisciplinaires. La secrétaire de la capacité qui jouait un rôle très important de tuteur social au téléphone et à l'accueil des cours, voit son rôle maintenu mais plutôt via le téléphone et le courriel en dehors des courtes périodes de présentiel.

Nous avons envisagé la pérennité du dispositif sur l'aspect institutionnel et pédagogique. Pour ce diplôme, la logique de service publique a été plutôt réaffirmée. Les inscriptions ont été maintenues au tarif du présentiel et l'UFR assure sur son budget le surcoût (tutorat) occasionné par le succès de la formation.

La plate-forme initialement utilisée (web-ct) a été remplacée par une plate-forme libre de droit. La migration sur Moodle s'est faite sans problème majeur. La formation assurée par le STIC auprès des enseignants et des apprenants a bien sûr été adaptée à ce nouvel environnement et la première rentrée vient de se faire sans difficulté. Pour pérenniser les contenus, les ressources numériques passent progressivement sur un LCMS (Scenari développé par l'UTC).

Actuellement, les équipes pédagogiques restent stables. Nous avons des enseignants militants, convaincus, conscients des difficultés de l'accompagnement et soucieux de mener à bien leur mission. Nous aurons dans quelques années à prévoir des changements dans l'équipe professorale (départ à la retraite). Il nous faut dès à présent anticiper ces changements.

Le passage à l'enseignement numérique a-t-il modifié notre cible ? Y a-t-il eu ouverture à un nouveau public plus autonome et, au contraire, fermeture à nos apprenants issus de notre bassin de recrutement initial? Nous n'aurons probablement jamais de réponses à ces questions. Il aurait fallu, pour ce faire, disposer d'études antérieures. Si nous avons gardé une bonne partie de notre public initial, il est clair qu'il y a pour l'apprenant 2 avantages certains mais difficiles à quantifier :

- l'apprentissage de la maitrise d'un outil informatique et d'une plate-forme collaborative. Démystifier un tel outil paraît un atout fondamental pour les apprenants même si au bout du compte, ils n'ont rien appris en droit,

- l'apprentissage réussi ou raté, mais au moins : tenter de l'autonomie.

Il est certain que le public n'est plus tout à fait le même. Le niveau est bien supérieur. Beaucoup d'apprenants sont déjà bacheliers. Pour eux, il s'agit probablement d'une recherche de compétences et non d'un diplôme. Certains recherchent des compétences pour un type de droit.

Il nous paraît en tout cas indispensable de mieux connaître notre public pour faire évoluer efficacement cette formation et rendre service au plus grand nombre. C'est pourquoi nous souhaitons mettre en place des enquêtes en début et en fin d'année pour mieux apprécier l'adéquation entre les objectifs des apprenants et les 
résultats. Nous espérons ainsi pouvoir disposer d'indicateurs utiles à la fois pour ce dispositif et pour d'autres formations à distance.

Une meilleure connaissance de ce contexte pourrait à terme nous aider à prendre des décisions en matière de collaborations inter-établissements. Cette question s'est d'ailleurs déjà posée dans deux circonstances (co-habilitation avec la Polynésie française, mutualisation au sein de l'Université Numérique Thématique juridique UNJF)

En plus de l'apprentissage de la maîtrise d'un outil informatique et d'une plateforme collaborative, l'expérience menée avec ce diplôme nous a incités à reproduire la modularisation de l'enseignement, en particulier dans le domaine de la formation professionnelle. La transformation de la maquette en modules sur des laps de temps plus courts (au lieu d'un étalement des enseignements sur l'année) devrait à terme répondre à des besoins de professionnels qui, tout en travaillant, pourront pendant 3-4 mois dégager un peu de temps pour acquérir une compétence dans une spécialité disciplinaire. On pourrait ainsi imaginer, au-delà de la capacité en droit, de développer, sur le même principe, des modules de formation sur 3-4 mois (comme pour le $\mathrm{C} 2 \mathrm{i}$ ) dans une discipline à des niveaux différents (initiation, perfectionnement...)

Cette hypothèse se pose aussi sur l'évolution du DAEU que nous mettons en ligne suivant les mêmes procédures. Il y a actuellement des matières obligatoires et des matières optionnelles. La formation pourrait être « élargie» vers des remises à niveau plus variées.

\section{Conclusion}

La gestion de ce projet a été une expérience très riche d'enseignements pour toutes nos équipes. Les premiers résultats obtenus sont très encourageants. Il reste à les vérifier sur le long terme. Conscients des écueils potentiels surtout compte tenu du contexte particulier de ce public, nous allons, avec le soutien d'experts, suivre avec la plus grande attention son évolution et tout particulièrement, le profil des apprenants, l'accompagnement pédagogique, les résultats académiques et autres...

Il est également primordial d'engager une pérennisation du dispositif tant au niveau de l'équipe enseignante que de la structure d'accompagnement.

Pour contribuer à assurer cette pérennité, les projets UVPL sont habituellement le fruit de collaborations inter-établissements. Compte tenu du désintérêt pour cette formation, cette mutualisation n'a pas été possible à la mise en place du dispositif mais nous souhaitons très vivement qu'elle s'ouvre à nos partenaires institutionnels pour lui assurer cette pérennité.

Sans le STIC, ce dispositif n'aurait probablement pas vu le jour. Il apparaît évident, aux yeux de l'équipe enseignante, que la cellule d'ingénierie pédagogique a largement contribué au succès du dispositif. Ces nouvelles compétences doivent, à 
terme, être reconnues et pérennisées dans nos établissements. Elles doivent également être encadrées et encouragées par des équipes de recherche pour mieux concevoir ces dispositifs et tout particulièrement les dispositifs d'accompagnement des apprenants qui restent, pour nous, le point stratégique de ces formations.

Révolution pédagogique et culturelle dans la composante de Droit, c'est aussi une révolution pour l'ensemble des enseignants-chercheurs de nos établissements qui, pour la plupart, ont besoin de «voir » des expériences concrètes de DFOAD avant de prendre en compte tout l'intérêt de ce type d'enseignement, lequel sera probablement la condition du succès de l'indispensable formation tout au long de la vie. 